Proc. of the 15th Int. Workshop on Slow Positron Beam Techniques and Applications, Prague, September 2-6, 2019

\title{
On the Reconstruction of the Mean Positron Lifetime Obtained from the Sequential Chemical Etching Procedure
}

\author{
J. DRYZEK* \\ Institute of Nuclear Physics, Polish Academy of Sciences, PL-31342 Krakow, Poland
}

\begin{abstract}
A method of reconstructing the actual depth profile of the mean positron lifetime obtained from the sequential chemical etching procedure has been proposed. In this procedure, the measured positron mean lifetime values as a function of depth are used to calculate the actual mean positron lifetime values at various depths. Two examples show the use of this method. The first one reconstructs the actual mean positron lifetime distribution in molybdenum subjected to friction. The second one reconstructs distribution in a silicon wafer irradiated with $167 \mathrm{MeV} \mathrm{Xe} \mathrm{X}^{26+}$ ions. These examples show this method's usefulness in detecting properties in subsurface zones generated by various surface treatments.
\end{abstract}

DOI: 10.12693/APhysPolA.137.196

PACS/topics: positron annihilation, ion implantation, friction, defect depth profiling

\section{Introduction and motivation}

The surface treatment is a common technological process to modify its properties. A good example is the ion implantation process that modifies the electrical properties in a layer of semiconductors adjacent to an irradiated surface. The sandblasting process is important for completing the production of commercial metallic products. However, the surface treatment leads to changes on the surface and below, for example, in the friction process. Structural changes in the region below the treated surface are rarely recognized and controlled. Also, theoretical predictions of such changes, for example, the depth distribution response caused by applied strain, can only be described in limited cases only. Positron annihilation spectroscopy (PAS) can be useful to study processes occurring below the surface.

PAS has been used for decades to study open volume defects, including defects' depth distribution, especially by use of slow positron beam technique $[1,2]$. However, conventional techniques based on positrons emitted from ${ }^{22} \mathrm{Na}$ can be used also successfully for investigation to detect depth distribution. The latter allows detecting defects at much larger depths than with slow positron beam technique, where a range of positrons is limited to only around $2 \mu \mathrm{m}$. For this purpose, the following procedure is used for many years [3]. A positron source enveloped in a kapton film is placed on the surface of a sample and the positron annihilation characteristics, for example, the mean positron lifetime are measured. This and also annihilation line shape parameter are sensitive to the defect concentration. After the measurements the sample is etched, for instance in acid to remove a layer of specific and controlled thickness and the

*corresponding author; e-mail: jerzy.dryzek@ifj.edu.pl measurement is repeated. Sequential etching and measurements of the positron annihilation characteristics allow obtaining their dependence on the etched thickness, i.e., the depth starting with the initial surface, which was exposed to the treatment. The experimental history shows that the etching does not introduce new defects detected by PAS. This procedure which we call the sequential chemical etching procedure determines defects distribution as a function of depth below the surface.

Since this is a destructive procedure, the sample is destroyed during measurement. Nevertheless, long practice indicates that the depth distribution profiles are reproducible, however, this procedure allows us to detect defects down to the depths to $500 \mu \mathrm{m}$. Recent studies have shown that it is possible to successfully determine the distribution generated also at shallow depth of about $10 \mu \mathrm{m}$, like in the case of defects caused by implantation of swift ions [4]. However, the question arises whether the shallow distributions are not distorted by the positron implantation profile. It should be remembered that the average depth of penetration of positrons emitted from ${ }^{22} \mathrm{Na}$ is about several dozen $\mu \mathrm{m}$, so it is much greater than the total depth of changes caused by implantation of swift ions. As a result, the measured characteristic is averaged over the positron implantation profile, which is also a function of depth.

In this report, we describe the method of reconstructing the actual characteristic measured as the function of depth obtained in the etching procedure described above. Such reconstruction is important because, e.g., the actual mean positron lifetime can be useful for calculating other properties, such as the depth profile of strain distribution that is important for engineering applications. An example of using this reconstruction to determine the distribution of defect depth in tribolayer in molybdenum, and the distribution of depth of damage caused by fast $167 \mathrm{MeV} \mathrm{Xe} \mathrm{X}^{26+}$ ions in silicon wafer will be presented. 


\section{The theoretical approach}

Assume that after etching a layer with a thickness of $z$, the measured value of the mean positron lifetime on the surface is denoted as: $\bar{\tau}_{m}(z)$. This value is related to the actual mean positron lifetime at depth $\xi: \bar{\tau}(\xi)$ as follows [5]:

$$
\bar{\tau}_{m}(z)=\int_{z}^{\infty} p(\xi-z) \bar{\tau}(\xi) \mathrm{d} \xi
$$

where $p(z)$ is the implantation profile of positrons emitted from the ${ }^{22} \mathrm{Na}$ isotope. A similar relationship can be written for another measured characteristic, i.e., the annihilation line shape parameter called the $S$-parameter

$$
S_{m}(z)=\int_{z}^{\infty} p(\xi-z) S(\xi) \mathrm{d} \xi .
$$

At this stage, let us assume that the positron implantation profile is expressed by the exponential decay function as follows:

$$
p(z)=\mu \exp (-\mu z),
$$

where $\mu$ is the linear absorption coefficient for positrons. This profile is normalized to unity. Equation (1) can be then expanded in the following series:

$$
\begin{gathered}
\bar{\tau}_{m}(z)=\int_{z}^{\infty} p(\xi-z), \bar{\tau}(\xi) \mathrm{d} \xi \simeq \\
\int_{z}^{z+\Delta z} \ldots+\int_{z+\Delta z}^{z+2 \Delta z} \ldots+\int_{z+2 \Delta}^{z+3 \Delta z} \ldots+\ldots,
\end{gathered}
$$

where $\Delta z$ represents the thickness of the etched layer at each step. The first term in this series can be approximated as follows:

$$
\begin{aligned}
& \int_{z}^{z+\Delta z} p(\xi-z) \bar{\tau}(\xi) \mathrm{d} \xi \simeq \\
& \frac{1}{2} \Delta z[p(0) \bar{\tau}(z)-p(\Delta z) \bar{\tau}(z+\Delta z)]= \\
& w\langle\bar{\tau}(z: z+\Delta z)\rangle-\frac{1}{2} w^{2} \bar{\tau}(z+\Delta z),
\end{aligned}
$$

where $w=\Delta z \mu$ and $\langle\bar{\tau}(z: z+\Delta z)\rangle$ represents the average value of the actual mean positron lifetime $\bar{\tau}$ at a depth range from $z$ to $z+\Delta z$. Assuming that $w \ll 1$ we can neglect the second term with $w^{2}$ in Eq. (5). Repeating this for other terms in Eq. (4) we obtain the following equation:

$$
\begin{aligned}
& \bar{\tau}_{m}(z) \simeq w\langle\bar{\tau}(z: z+\Delta z)\rangle \\
& \quad+w \sum_{i=1} \mathrm{e}^{-w i}\langle\bar{\tau}(z+i \Delta z: z+(i+1) \Delta z)\rangle .
\end{aligned}
$$

Subsequently, we write a similar equation but for the measured mean positron lifetime after etching a layer of thickness $\Delta z$ :

$$
\begin{gathered}
\bar{\tau}_{m}(z+\Delta z)=\int_{z+\Delta z}^{\infty} p(\xi-z-\Delta z) \bar{\tau}(\xi) \mathrm{d} \xi \simeq \\
w \sum_{i=1} \mathrm{e}^{-w(i-1)}\langle\bar{\tau}(z+i \Delta z: z+(i+1) \Delta z)\rangle .
\end{gathered}
$$

After subtracting Eq. (7) and Eq. (6) we obtain the following equation for the average value of the actual mean positron lifetime at the depth range from $z$ to $z+\Delta z$ :

$\langle\bar{\tau}(z: z+\Delta z)\rangle \simeq \bar{\tau}_{m}(z+\Delta z)+\frac{\bar{\tau}_{m}(z)-\bar{\tau}_{m}(z+\Delta z)}{w}$.

For $\Delta z \rightarrow 0$ this is sought to be the value of the actual mean positron lifetime at the depth of $z$. In further considerations, the results in Eq. (8) will be treated as the actual positron lifetime at the depth of $z$. In Eq. (8) the $\Delta z$ is the thickness of the etched layer, however, in practice, this value can be varied at each step then this equation can be extended as follows:

$\left\langle\bar{\tau}\left(z: z+\Delta z_{k}\right)\right\rangle \simeq \bar{\tau}_{m}\left(z+\Delta z_{k}\right)+\frac{\bar{\tau}_{m}(z)-\bar{\tau}_{m}\left(z+\Delta z_{k}\right)}{w_{k}}$,

where $w_{k}=\mu \Delta z_{k}$ and $\Delta z_{k}$ is the thickness of an etched layer at every step.

A small value, i.e., $w$ less than the unity desired in Eq. (6) is placed in the denominator in Eq. (8). This significantly affects the accuracy of the average value of the actual mean positron lifetime. Also, $w$ depends on two values determined experimentally. The etched layer thickness in a common experiment can be determined within the accuracy of $\pm 1 \mu \mathrm{m}$, if a digital micrometer screw is used. The approximated value of the linear absorption coefficient $\mu$ can be another source of uncertainty.

The Monte Carlo simulations, as well as experiments carried out by other authors [6], show that the positron implantation profile from the $\beta^{+}$decay isotope has a much more complex form than that expressed in Eq. (3). The authors suggest a better description of this profile can be expressed as follows [7]:

$$
p(z)=N \mu \begin{cases}\exp (-2 \mu z), & z \leq d, \\ \exp (-\mu(z+d)), & z>d,\end{cases}
$$

where $N=2 /[1+\exp (-2 \mu d)]$ is the normalized constant and $d \simeq 0.5 / \mu$. Thus in Eqs. (8) and (9), we should use: $w=4 \mu /\left(1+\mathrm{e}^{-1}\right) \Delta z=2.924 \mu \Delta z$, when $\Delta z<d$ in the experiment and $w=2 \mu /\left(1+\mathrm{e}^{-1}\right) \Delta z=1.462 \mu \Delta z$, when $\Delta z>d$.

Other authors suggest using different functions to describe the implantation profile, but they require more parameters than one, as in Eq. (10) [8, 9]. However, at this stage, the Monte Carlo simulations can be used instead of experimental methods to determine them. The value of the linear absorption coefficient can be calculated using an empirical formula: $\mu\left[\mathrm{cm}^{-1}\right]=12.6 \rho Z^{0.17} / E_{\max }^{1.28}$, where $\rho$ is a density of material that is implanted by positron's in $\left[\mathrm{g} / \mathrm{cm}^{3}\right], Z$ is the atomic number, and $E_{\max }$ is the endpoint of the beta + spectrum, which in ${ }^{22} \mathrm{Na}$, is $0.545 \mathrm{MeV}$ [7]. 


\section{The application of the proposed method}

\subsection{Molybdenum exposed to the friction}

For many years, we used the sequential chemical etching procedure to study the distribution of positron lifetime characteristics in samples subjected to friction [3]. The damage region was observed even at a depth of hundreds of micrometers with a characteristic exponential decay as the depth increased. Here we show the example with pure molybdenum sample exposed to the dry sliding process.

A molybdenum sample of $99.95 \%$ purity purchased from Goodfellow, used in our experiment had the shape of a disc with a diameter of $10 \mathrm{~mm}$ and a thickness of $2 \mathrm{~mm}$. Initially, two discs were annealed in a vacuum $\left(10^{-5}\right.$ Torr $)$ at $1010^{\circ} \mathrm{C}$ for $1 \mathrm{~h}$, and then slowly cooled to room temperature. The samples were etched in the 10:10:2 volume mixture of $\mathrm{H}_{2} \mathrm{O}: \mathrm{HNO}_{3}: \mathrm{HF}$ to remove a layer of the surface about $50 \mu \mathrm{m}$ thick.

The present work used digital positron lifetime spectrometer purchased from TechnoAP with two photomultipliers: H3378-50 coupled with $\mathrm{BaF}_{2}$ scintillators. The time resolution of the spectrometer was about $170 \mathrm{ps.} \mathrm{The}$ isotope ${ }^{22} \mathrm{Na}$ was used as the positron source. It was enveloped into a $7 \mu \mathrm{m}$ thick kapton foil, with the activity of about $20 \mu \mathrm{Ci}$. In molybdenum, the linear absorption coefficient for these positrons is about $527 \mathrm{~cm}^{-1}$ from the empirical formula. All spectra were deconvoluted using LT code [10].

The measurement of positron lifetime revealed one component that equals $115(0.7)$ ps in the virgin sample. This value aligns with the values for bulk lifetime reported in the literature: 115 ps in Ref. [11], 120 ps in Ref. [12], and 118-120 ps in Ref. [13]. Then the virgin sample was mounted in a tribotester and its surface was exerted with a load of $25 \mathrm{~N}$ onto a rotating disk for $1 \mathrm{~min}$. The rotating disc $50 \mathrm{~mm}$ in diameter was made of the high-speed steel (HS18 with hardness about 670 HV0.1). The speed of the disc relative to the surface of the sample was about $15 \mathrm{~cm} / \mathrm{s}$. The test was carried out in air for one minute.

The measurement of the positron lifetime spectrum directly at the worn surface revealed two-lifetime components. The value of the first one was equal to 126(4) ps and the second one 237(11) ps with the intensities of $75(3) \%$ and 25(3) \%, respectively. This indicates the presence of dislocations and small vacancy clusters consisting of about two vacancies $[14,15]$. With the increasing depth, the intensity of the second lifetime component decreases. The further analysis, however, is based on the mean positron lifetime, calculated from the obtained data. The determined mean lifetime values in function of depth are depicted in Fig. 1. It decreases exponentially with the depth and at the depth of about $75 \mu \mathrm{m}$ reaches the bulk value, tagged by the hatched region. This is the total depth of the subsurface zone generated during the dry sliding test. It is the shortest range as we observed up to now. The solid line in Fig. 1 represents the

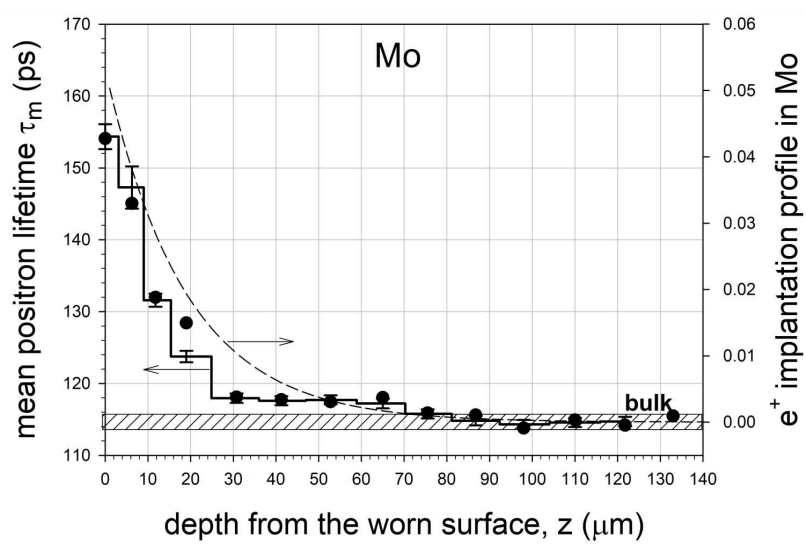

Fig. 1. The measured mean positron lifetime as the function of depth for pure molybdenum sample subjected to the friction, closed circles. The solid line represents the results of reconstruction of the actual mean positron lifetime using Eq. (9) and the dashed line the implantation profile of ${ }^{22} \mathrm{Na}$ positrons in molybdenum (right axis), see Eq. 10. The hatched region tags the value of positron lifetime resolved for a well-annealed sample.

results of reconstruction of the mean positron lifetime obtained using Eq. (9). One can notice that this dependence aligns with the experimental points, no significant deviation is observed. The dashed line in Fig. 1 represents the positron implantation profile in molybdenum (right axis), see Eq. 10. The reconstruction, in this case, is not necessary, since the range of mean positron lifetime, solid line is comparable with the range of positrons, dashed line, in Fig. 1. However, in the case when the depth range of generated defects is much less than the range of positrons, as it is in the case of implantation of swift ions, the reconstruction will be required.

\subsection{Silicon irradiated with Xe ions}

The proposed reconstruction method has been applied to studies of the depth distribution of mean positron lifetime in pure bismuth irradiated with swift $\mathrm{Xe}^{26+}$ ions [16]. This encouraged us to conduct the studies in a silicon polished wafer (100) obtained by a floating zone method. The implantation of the wafer was performed at IC-100 cyclotron at Flerov Laboratory of Nuclear Reactions at Joint Institute for Nuclear Research (JINR) in Dubna, Russia. $\mathrm{Xe}^{26+}$ heavy ions with an energy of $167 \mathrm{MeV}$ and dose of $5 \times 10^{12} \mathrm{ions} / \mathrm{cm}^{2}$ were implanted into the wafer, with no additional treatments performed before. The samples were etched in the 4:1 volume mixture of $\mathrm{HNO}_{3}: \mathrm{HF}$ acids. The projectile range of this ion in the silicon according to the SRIM code is about $21 \mu \mathrm{m}$ [17]. In the all measured positron lifetime spectra, only a single lifetime component was resolved. Before implantation, the value of 208(1) ps was found and it corresponds to the data reported in the literature [18]. The measurement at the entrance surface after implantation revealed that the positron lifetime increased 


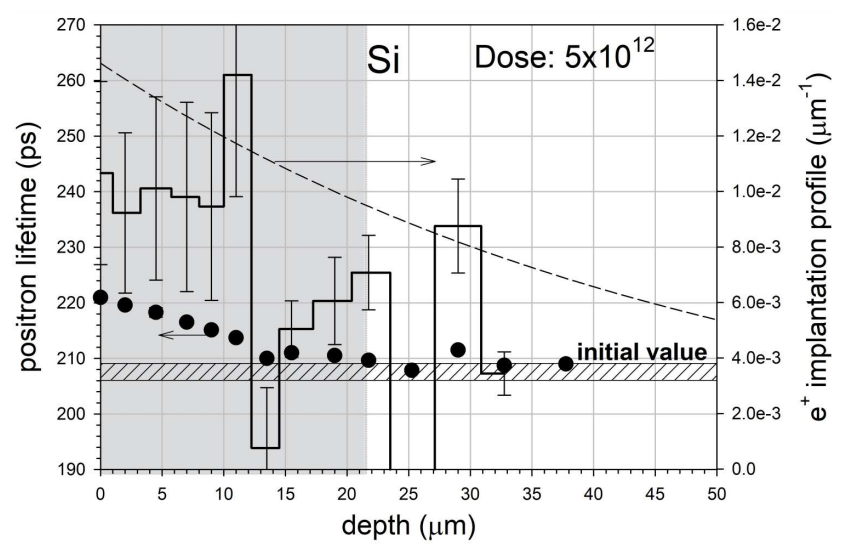

Fig. 2. The measured mean positron lifetime as the function of depth for silicon wafer subjected to irradiation with $167 \mathrm{MeV} \mathrm{Xe}{ }^{26+}$ ions, closed circles. The solid line represents the results of reconstruction of the actual mean positron lifetime using Eq. (9) and the dashed line the implantation profile of ${ }^{22} \mathrm{Na}$ positrons in silicon, see Eq. 10. The gray rectangular tags the implanted layer, where ions are traveling until they slow down and stop completely. The hatched region represents the value of positron lifetime resolved for the sample before implantation.

to about $221(1) \mathrm{ps}$. In the literature similar value is reported in undoped float zone silicon after $3 \mathrm{MeV}$ electron irradiation at $20 \mathrm{~K}$ with a dose of $10^{15} \mathrm{~cm}^{-2}$ [19].

After etching the lifetime value decreases linearly, and at the depth of about $20 \mu \mathrm{m}$, the "initial value" was reached, as shown in Fig. 2, where the closed circles represent the resolved values as the function of the depth starting with the entrance surface. The solid line in Fig. 2 represents the reconstruction of the positron lifetime using Eq. (9) to the experimental points. It is visible that up to the depth of about $12 \mu \mathrm{m}$ the actual value is almost constant and equal to about 240 ps and then decreases with the increase in depth. This is contrary to the experimental values (closed circles) which show a linear decrease with the depth. The 250 ps value was reported as the positron lifetime the neutral mono vacancy in silicon [20]. For silicon, the linear absorption coefficient is equal to $100 \mathrm{~cm}^{-1}$, and the positron implantation profile is presented in Fig. 2 as the dashed line, see Eq. 10. The range of positrons is much longer than the range of damage induced by implantation with $\mathrm{Xe}^{26+}$ ions, and this causes significant differences. One can note large scatter of the actual values obtained. This arises from the fact that an average $w$ value is equal to about 0.06 , and such a small value is in the denominator in Eq. (9). Nevertheless, the fact that the actual values of the mean positron lifetime remain almost constant within the layer directly affected by the ions tagged by a gray region in Fig. 2 is an interesting result. This is a contradiction to predicting calculations of SRIM code [17], which indicate that larger vacancy concentration is at the end of the ion track, i.e., near the projectile range. The experimental results suggest that beyond the projectile range we did not find the presence of defects which would be indicated by an increase of the mean positron lifetime. Thus no "long range effect", as reported by other authors [21] was observed, presumably the applied dose is too small to induce this effect. This example shows us that reconstruction may be necessary when using a sequential chemical etching procedure for testing implanted layers.

\section{Conclusions}

The method for reconstruction of the actual value of mean positron lifetime from the experimental values obtained from the sequential chemical etching procedure was proposed. The method is suitable for reconstruction of results of experiments when the range of positrons significantly differs in the range of generated defects, as it is in the case of implantation of swift ions. This was shown on the example of silicon wafer implanted with $167 \mathrm{MeV} \mathrm{Xe} \mathrm{X}^{26+}$ ions. The actual mean positron lifetime obtained is almost constant to the depth of about $12 \mu \mathrm{m}$, and then decreases to the initial value. This result is in contradiction to the results of the Monte Carlo simulation performed by SRIM code. Approximately, the range of damage region induced by swift ions implantation corresponds with the projectile range, and no "long range effect" was found. When the distribution of the defect range was comparable to the positron range, the actual and measured value align pretty well. This occurs when defects arise as a result of friction or other mechanical processes.

\section{Acknowledgments}

The author expresses their gratitude to V.A. Skuratov for his technical help and assistance in ion irradiation and P. Horodek for additional technical assistance.

\section{References}

[1] R. Krause-Rehberg, H.S. Leipner, in: Positron Annihilation in Semiconductors, Springer, Berlin 1999.

[2] A. Van Veen, H. Schut, P.E. Mijnarends, in: Positron Beams and Their Applications, Ed. P.G. Coleman, World Sci., Singapore 2000, p. 191.

[3] J. Dryzek, E. Dryzek, R. Krause-Rehberg, F. Redman, Tribol. Lett. 11, 121 (2001).

[4] P. Horodek, J. Dryzek, V.A. Skuratov, Vacuum 138, 15 (2017).

[5] J. Dryzek, Appl. Phys. A 114, 465 (2014).

[6] K. Płotowski, T.J. Panek, J. Kansy, Nuovo Cim. D 10, 933 (1988).

[7] J. Dryzek, D. Singleton, Nucl. Instrum. Methods Phys. Res. B 252, 197 (2006).

[8] L.Yu. Dubov, Yu.A. Akmalova, S.V. Stepanov, Yu.V. Funtikov, Yu.V. Shtotsky, Acta Phys. Pol. A 132, 1482 (2017).

[9] J. Dryzek, J. Sieracki, Nucl. Instrum. Methods Phys. Res. B 258, 493 (2007). 
[10] J. Kansy, LT ver. 10, LT Programs, 2010.

[11] T. Hyodo, B.T.A. McKee, A.T. Stewart, Radiat. Eff. 68, 77 (1982).

[12] B. Pagh, H.E. Hansen, B. Nielsen, G. Trumpy, K. Petersen, Appl. Phys. A 33, 255 (1984).

[13] Z. Zhiyong, C. Keqin, C. Jiachao, X. Ronghui, W. Zhiguang, Nucl. Instrum. Methods Phys. Res. B 61, 72 (1991).

[14] B. Nielsen, et al., in: Positron Annihilation, Eds. P.C. Jain, R.M. Singru, K.P. Gopinathan, World Sci. Singapore 1985, p. 497.

[15] S.N. Khanna, B.K. Rao, P. Jena, D. Esterling, M.J. Puska, Phys. Rev. B 37, 6 (1988).

[16] J. Dryzek, K. Siemek, Appl. Phys. A 125, 85 (2019).
[17] J.F. Ziegler, The Stopping and Range of Ions in Solids, Pergamon, New York 2003.

[18] Y. Shirai, M. Mizuno, H. Araki, in: Encyclopedia of Materials: Science and Technology, 2nd ed., Eds. K.H.J. Buschow, R.W. Cahn, M.C. Flemings, B. Ilschner, E.J. Kramer, S. Mahajan, P. Veyssière, 2005, p. 1.

[19] S. Mäkinen, M.J. Puska, Phys. Rev. B 40, 12523 (1989).

[20] M.J. Puska, C. Corbel, Phys. Rev. B 38, 9874 (1988).

[21] Yu.P. Sharkeev, E.V. Kozlova, Surf. Coat. Technol. 158, 219 (2002) 